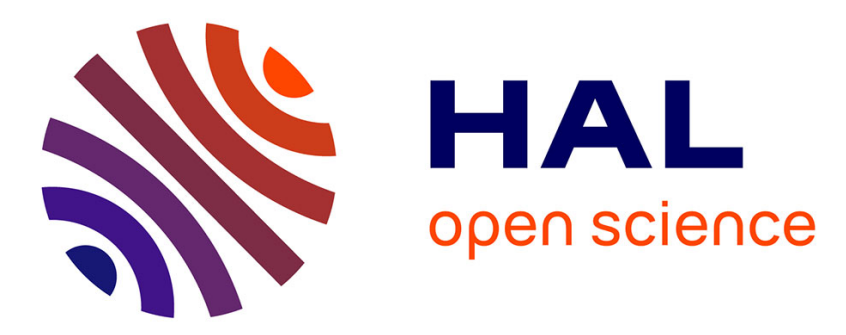

\title{
Finite-sample density and its small sample asymptotic approximation
}

Jana Jurečková, Radka Sabolová

\section{To cite this version:}

Jana Jurečková, Radka Sabolová. Finite-sample density and its small sample asymptotic approximation. Statistics and Probability Letters, 2011, 81 (8), pp.1311. 10.1016/j.spl.2011.03.034 . hal00753941

\section{HAL Id: hal-00753941 \\ https://hal.science/hal-00753941}

Submitted on 20 Nov 2012

HAL is a multi-disciplinary open access archive for the deposit and dissemination of scientific research documents, whether they are published or not. The documents may come from teaching and research institutions in France or abroad, or from public or private research centers.
L'archive ouverte pluridisciplinaire HAL, est destinée au dépôt et à la diffusion de documents scientifiques de niveau recherche, publiés ou non, émanant des établissements d'enseignement et de recherche français ou étrangers, des laboratoires publics ou privés. 


\section{Accepted Manuscript}

Finite-sample density and its small sample asymptotic approximation

Jana Jurečková, Radka Sabolová

PII:

S0167-7152(11)00125-8

DOI:

10.1016/j.spl.2011.03.034

Reference: $\quad$ STAPRO 5969

To appear in: Statistics and Probability Letters

Received date: 12 January 2011

Revised date: 24 March 2011

Accepted date: 26 March 2011

Please cite this article as: Jurečková, J., Sabolová, R., Finite-sample density and its small sample asymptotic approximation. Statistics and Probability Letters (2011), doi:10.1016/j.spl.2011.03.034

This is a PDF file of an unedited manuscript that has been accepted for publication. As a service to our customers we are providing this early version of the manuscript. The manuscript will undergo copyediting, typesetting, and review of the resulting proof before it is published in its final form. Please note that during the production process errors may be discovered which could affect the content, and all legal disclaimers that apply to the journal pertain. 


\title{
Finite-sample density and its small sample asymptotic approximation
}

\author{
Jana Jurečková, Radka Sabolová \\ Department of Probability and Statistics, MFF UK, Charles University, \\ Sokolovská 83, CZ-186 75 Prague 8, Czech Republic
}

\begin{abstract}
To derive the exact density of a statistic, which can be intractable, is sometimes a difficult problem. The exact densities of estimates of the shift or regression parameters can be derived with the aid of score functions. Moreover, extremely accurate approximations can be obtained by the small sample asymptotics, based on the saddlepoint method. It is of interest to compare these two approaches, at least for small samples. We numerically compare the exact densities of estimates of the shift parameter with their small sample approximations for various parent distributions of the data. For some distributions both methods are in surprising concordance even under very small samples.
\end{abstract}

Keywords: finite-sample density, small sample asymptotics, saddlepoint approximation, score function

2010 MSC: 62E15, 62E17, 65C60

\section{Introduction}

Many estimators have asymptotic normal distribution, which is lighttailed. However, the asymptotic distribution approximates well the central

\footnotetext{
ॠNesearch of J. Jurečková was supported by the Grant GAČR201/09/0133 and by Research Projects MSM 0021620839 and LC 06024.

触The work of R. Sabolová was supported by the grant SVV 261315/2010.

Email addresses: jurecko@karlin.mff.cuni.cz (Jana Jurečková), sabolova@karlin.mff.cuni.cz (Radka Sabolová)

URL: http://www.karlin.mff.cuni.cz/〜 jurecko (Jana Jurečková)
} 
part of the true distribution, but not so well its tails. An estimator can be heavy-tailed under a finite number of observations, even the robust one, provided the parent distribution is heavy-tailed. We should be also cautious when using the asymptotic tests, because their critical regions are based on the high quantiles of the asymptotic distribution, while the finite-sample distribution of the test criterion can be heavy-tailed. Hence, before taking a recourse to the asymptotics, we should first analyze the finite-sample behavior of an estimator or a test, whenever possible.

To derive the exact density of a statistic is often a difficult problem, and the finite sample density of a statistic can be intractable. One possible way of expressing the finite-sample density of a statistic is to use its score function, which is a conditional expectation of the parent score function, given the pertaining statistic. This method was used by Jurečková (1999), (2010) and Jurečková and Milhaud (2003) for a general statistic and for the regression quantile.

Extremely accurate approximations for the density of a statistic and for the $p$-values of a test criterion, even under a very small sample size, yields the saddlepoint method or the saddlepoint approximation. Saddlepoint approximations were introduced to statistics by Daniels (1954) and further elaborated by Hampel (1973) and by Field and Hampel (1982); we also refer to Barndorff-Nielsen and Cox (1979). The saddlepoint density approximation can be considered as the best Edgeworth approximation at each point in the support of the distribution. However, the computations of such approximations became feasible only rather recently. This technique, also known as the small-sample asymptotics, was developed by Field and Ronchetti (1990) for a class of estimators, including the robust ones. Let us refer also to Huzurbazar (1999), who illustrates the practical use of the saddlepoint approximation on the sample mean and other statistics for the sample size $n=1$, and to references cited in his article. Among more recent books we refer to Jensen (1995) and to Butler (2007). Both the exact finite-sample and the small sample asymptotic approximations of the density of a statistic are connected with an intensive computation, affected also by the computer rounding. Thus it is of interest to verify the effectiveness of both methods and to compare them numerically. This is done in the present paper on various special estimates of the shift parameter and on several parent distributions. In many cases, the outcomes of both methods are surprisingly close to each other. 


\section{Finite-sample density of equivariant estimators}

We are interested in the density of a statistic in its whole scope, not only in the center. This is always a difficult problem, and generally the finite sample density of a statistic does not have a simple form. Under some conditions we can find a density of a statistic in the model with a shift or regression parameters. Let $X_{1}, \ldots, X_{n}$ be a sample from the distribution with distribution function $F(x-\theta)$ such that $F$ has a continuously differentiable density $f$ and finite Fisher information. Let $S_{n}=S_{n}\left(X_{1}, \ldots, X_{n}\right)$ be a statistic whose distribution function $H_{\theta}(s)$ is continuously differentiable in $\theta$. Then we have the following identity for the derivative of $H_{\theta}(s)$ in $\theta$ (see Jurečková (1999)):

$$
\begin{aligned}
\frac{\partial H_{\theta}(s)}{\partial \theta} & =\int_{S\left(x_{1}, \ldots, x_{n}\right) \leq s} \ldots \int \sum_{i=1}^{n}\left(-\frac{f^{\prime}\left(x_{i}-\theta\right)}{f\left(x_{i}-\theta\right)}\right) \prod_{k=1}^{n} f\left(x_{k}-\theta\right) d x_{1} \ldots d x_{n} \\
& =\mathbb{E}_{\theta}\left[\sum_{i=1}^{n}\left(-\frac{f^{\prime}\left(x_{i}-\theta\right)}{f\left(x_{i}-\theta\right)}\right) \cdot I\left[S\left(X_{1}, \ldots, X_{n}\right) \leq s\right]\right] .
\end{aligned}
$$

Let, especially, $T_{n}$ be a translation equivariant estimator of $\theta$ and let $g_{\theta}(t)$ be its density. A possible finite-sample expression for density $g_{\theta}(t)$ we get from (1); it will further enable to study various properties of $T_{n}$ :

$$
\begin{aligned}
& g_{\theta}(t)=\int_{T\left(x_{1}, \ldots, x_{n}\right) \leq t} \cdots \int \sum_{i=1}^{n} \frac{f^{\prime}\left(x_{i}-\theta\right)}{f\left(x_{i}-\theta\right)} \prod_{k=1}^{n} f\left(x_{k}-\theta\right) d x_{1} \ldots d x_{n} \\
& =\mathbb{E}_{0}\left\{\sum_{i=1}^{n} \frac{f^{\prime}\left(X_{i}\right)}{f\left(X_{i}\right)} I\left[T\left(X_{1}, \ldots, X_{n}\right) \leq t-\theta\right]\right\} .
\end{aligned}
$$

If $T_{n}$ is a solution of the equation $\sum_{i=1}^{n} \psi\left(X_{i}-t\right)=0$ with monotone $\psi$, then $g(t)$ can be rewritten

$$
g_{\theta}(t)=\mathbb{E}_{0}\left\{\sum_{i=1}^{n} \frac{f^{\prime}\left(X_{i}\right)}{f\left(X_{i}\right)} I\left[\sum_{j=1}^{n} \psi\left(X_{j}-(t-\theta)\right) \leq 0\right]\right\} .
$$

Besides the distribution, the exact finite-sample moments of an estimate are of interest. An identity for the derivative of the $\nu$-th moment $\gamma^{(\nu)}(\theta)=$ $\mathbb{E}_{\theta}\left(T_{n}\right)^{\nu}$ of $T_{n}$ follows from Jurečková and Milhaud (2003):

$$
\dot{\gamma}^{(\nu)}(\theta)=\mathbb{E}_{0}\left[\left(T_{n}(\mathbf{X})+\theta\right)^{\nu}\left(-\sum_{i=1}^{n} \frac{f^{\prime}\left(X_{i}\right)}{f\left(X_{i}\right)}\right)\right] .
$$


It holds for all $\theta \in \mathbb{R}$, provided $\gamma^{(\nu)}(\theta)$ is finite and differentiable in $\theta ; \nu>0$ is not necessary an integer. The moment convergence of the $M$ - and $L$ estimates of $\theta$ as $n \rightarrow \infty$ (to the moments of their asymptotic normal distributions) was proved by Jurečková and Sen (1982) under some conditions on their weight functions. The identity (4) enables to compare the finite-sample moments with their limits.

\section{Small sample asymptotics}

Let $X_{1}, \ldots, X_{n}$ be i.i.d. observations with density $f(x, \theta)$. Consider an M-estimator $T_{n}$ of $\theta$, defined as a solution of the equation $\sum_{i=1}^{n} \psi\left(X_{i}, t\right)=0$. Denote its density as $f_{n}(t)$. An approximation of density of M-estimator is based on techniques derived for the mean (Field and Ronchetti (1990), Chapter 3$) ; T_{n}$ is expressed as a mean up to a certain order and then the saddlepoint approximation for the mean is used. Denote $D^{n}$ the $n$-th derivative of $\psi(x, \theta)$ with respect to $\theta$. The following assumptions on $\psi$ and $f(x, \theta)$ are required in order to develop the approximation.

A.1 The equation $\sum_{i=1}^{n} \psi\left(X_{i}, t\right)=0$ has a unique solution $T_{n}$ and the equation

$$
\int \psi(x, t) e^{\alpha(t) \psi(x, t)} f(x, \theta) \mathrm{dx}=0
$$

has a unique solution $\alpha(t)$.

A.2 There is an open subset $U \subset \mathbb{R}$ such that

(i) $F_{\theta}(U)=1$ for each $\theta \in \Theta$

(ii) $D \psi(x, \theta), D^{2} \psi(x, \theta), D^{3} \psi(x, \theta)$ exist.

A.3 For each compact $K \subset \Theta$

(i) $\sup _{\theta_{0} \in K} \mathbb{E}_{\theta_{0}}\left|D^{2} \psi\left(X, \theta_{0}\right)\right|^{4}<\infty$,

(ii) there is an $\varepsilon>0$ such that $\sup _{\theta_{0} \in K} \mathbb{E}_{\theta_{0}}\left(\max _{\left|\theta-\theta_{0}\right| \leq \varepsilon}\left|D^{3} \psi(X, \theta)\right|^{3}\right)<$ $\infty$.

A.4 $\mathbb{E}_{\theta_{0}} \psi\left(X, \theta_{0}\right)=0$ and $A\left(\theta_{0}\right)=\mathbb{E}_{\theta_{0}} D \psi\left(X, \theta_{0}\right) \neq 0$ for each $\theta_{0} \in \Theta$.

A.5 The functions $A(\theta)$ and $\mathbb{E}_{\theta_{0}}\left[D^{2} \psi(X, \theta)\right]^{2}$ are continuous on $\Theta$.

The following theorem summarizes the approximation of $f_{n}(t)$ (see Theorem 4.3 in Field and Ronchetti (1990)): 
Theorem 3.1. (Field and Ronchetti). If $T_{n}$ represents the solution of $\sum_{i=1}^{n} \psi\left(x_{i}, t\right)=0$ and $A .1$ - A.5 hold, then the density of $T_{n}$ admits the following asymptotic expansion:

$$
f_{n}\left(t_{0}\right)=\sqrt{\frac{n}{2 \pi}} c^{-n}\left(t_{0}\right)\left[\frac{A\left(t_{0}\right)}{\sigma\left(t_{0}\right)}+O\left(n^{-1}\right)\right]
$$

where $\alpha\left(t_{0}\right)$ is the solution of

$$
\begin{gathered}
\int \psi\left(x, t_{0}\right) e^{\alpha \psi\left(x, t_{0}\right)} f(x, \theta) \mathrm{dx}=0, \\
A\left(\theta_{0}\right)=\mathbb{E}_{\theta_{0}} \frac{\partial \psi\left(X, \theta_{0}\right)}{\partial \theta_{0}}, \\
c^{-1}\left(t_{0}\right)=\int e^{\alpha\left(t_{0}\right) f(x, \theta)} \mathrm{dx}, \\
\sigma^{2}\left(t_{0}\right)=E_{t_{0}} \psi^{2}\left(X, t_{0}\right)
\end{gathered}
$$

and $\mathbb{E}_{t_{0}}$ is expectation with respect to the conjugate density

$$
h_{t_{0}}(x)=c\left(t_{0}\right) e^{\alpha\left(t_{0}\right) \psi\left(x, t_{0}\right)} f(x) .
$$

Theorem 3.1 gives the following approximation of density $f_{n}$ :

$$
g_{n}\left(t_{0}\right)=\sqrt{\frac{n}{2 \pi}} c^{-n}\left(t_{0}\right) \frac{A\left(t_{0}\right)}{\sigma\left(t_{0}\right)} .
$$

This approximation will be computed for several estimates and will be compared with their exact densities.

\section{Numerical study}

The densities calculated from (2) are compared with their approximations (9) for three location estimators:

(i) The mean (M-estimator with $\psi(x)=x$ ).

(ii) The Huber M-estimator with

$$
\psi(x)=\left\{\begin{array}{ll}
x & :|x| \leq k \\
k \cdot \operatorname{sign}(x) & :|x| \geq k
\end{array},\right.
$$

where $k$ was set to 1.4 . 
(iii) The maximum likelihood estimator, i.e. the M-estimator with

$$
\psi(x)=-\frac{f^{\prime}(x)}{f(x)} .
$$

The calculation was made for the following parent distributions:

- Standard normal distribution $\mathrm{N}(0,1)$ with density

$$
f(x)=\frac{1}{\sqrt{2 \pi}} e^{-\frac{x^{2}}{2}} \text { and }-\frac{f^{\prime}(x)}{f(x)}=x .
$$

- Logistic distribution $\log (0,1)$ with density

$$
f(x)=\frac{e^{-x}}{\left(1+e^{-x}\right)^{2}} \text { and }-\frac{f^{\prime}(x)}{f(x)}=-\frac{1-e^{x}}{1+e^{x}} .
$$

- The Cauchy distribution $\mathrm{C}(0,1)$ with density

$$
f(x)=\frac{1}{\pi\left(1+x^{2}\right)} \text { and }-\frac{f^{\prime}(x)}{f(x)}=\frac{2 x}{1+x^{2}} .
$$

- The Student t-distribution with 3 degrees of freedom $t_{3}$ with density $f(x)=\frac{6 \sqrt{3}}{\pi\left(3+x^{2}\right)^{2}}$ and $-\frac{f^{\prime}(x)}{f(x)}=\frac{4 x}{3+x^{2}}$.

- The Student t-distribution with 5 degrees of freedom $t_{5}$ with density $f(x)=\frac{100 \sqrt{5}}{3 \pi\left(5+x^{2}\right)^{3}}$ and $-\frac{f^{\prime}(x)}{f(x)}=\frac{6 x}{5+x^{2}}$.

Various steps and regions of numerical integration depending on the shape of the distribution were considered for the specific densities. All simulations were coded in $\mathrm{C}$.

As the approximation of multidimensional integrals is quite time consuming, the densities using the formula (2) were evaluated for small values of $n$, namely the results are available only for $n \leq 4$ (and $n \leq 3$ for the Cauchy distribution).

When approximating the density of $T_{n}$ by formula (9), it is necessary to find $\alpha(t)$ solution of the equation

$$
\int \psi(x, t) e^{\alpha(t) \psi(x, t)} f(x, \theta) \mathrm{dx}=0 .
$$

For $\psi(x, t)=x-t$ (i.e. the mean) and $f(x)$ being density of $N(0,1)$ is $\alpha(t)=t$. For other distributions and estimators one can use the fact that $\alpha(t)=0$ always for $t=\theta$; that provides a good starting point for the NewtonRaphston method for other $t$ [however, the exception is $\psi(x, t)=x-t$ 

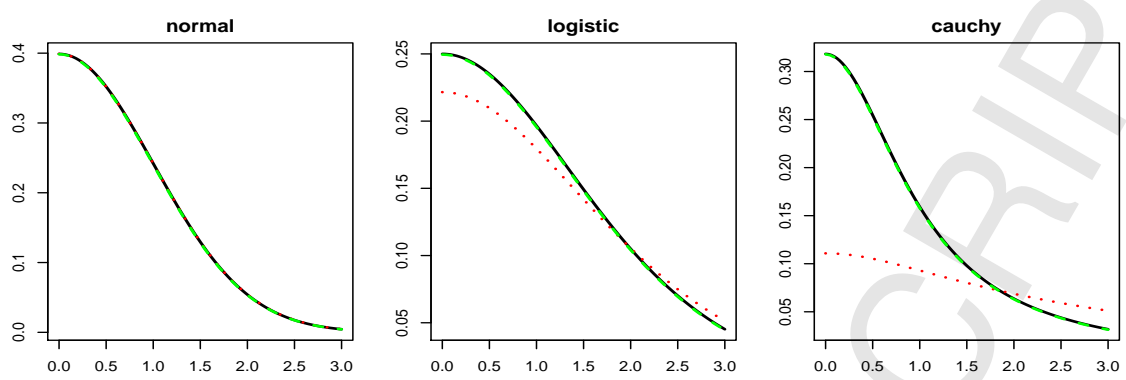

Figure 1: Density of the mean, $n=1$
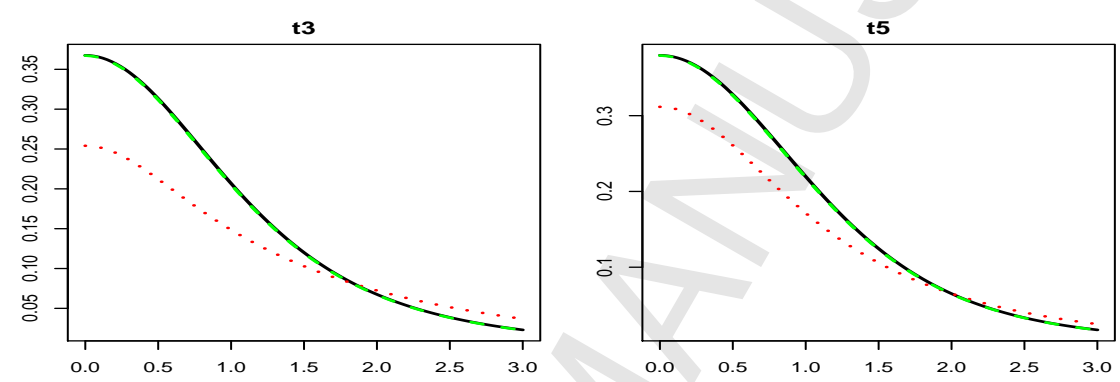

Figure 2: Density of the mean, $n=1$

combined with the Cauchy distribution]. Then $c, S$ and $A$ are approximated by numerical integration using formulas (7), (8) and (6) and then inputed into formula (9) for $f_{n}$.

When approximating mean for $n=1$ we get approximation of the original density $f$. Results of these approximation are presented in the Figures 1 and 2 , where exact density is drawn by solid line, saddle point approximation by dashed line and formula (2) by dotted line.

The outcomes of both methods are presented in the Figures 3 -16: The results based on formula (2) are drawn by dashed line, while those by formula (9) are drawn by dotted line. 

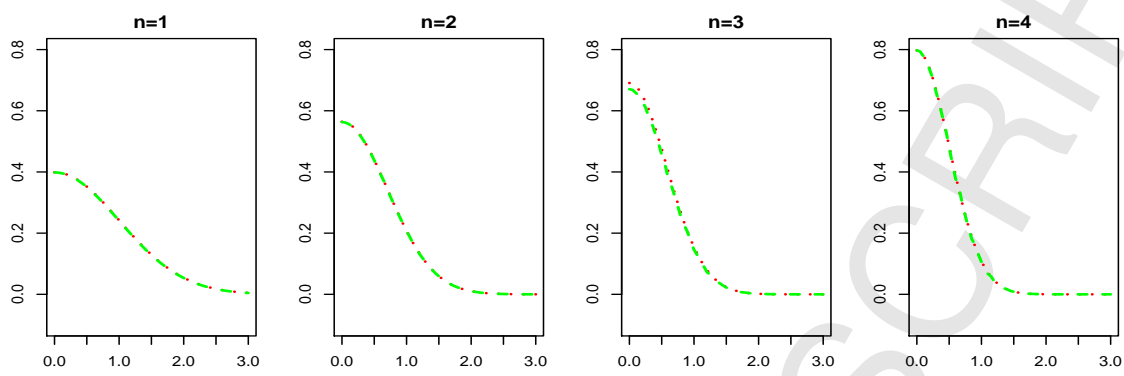

Figure 3: Density of the mean based on the sample from $\mathrm{N}(0,1)$
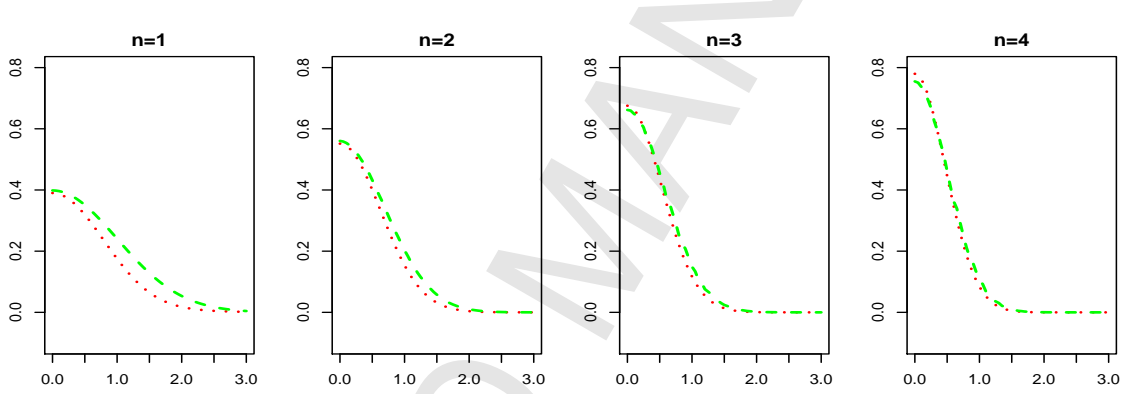

Figure 4: Density of the Huber estimator, $\mathrm{k}=1.4$, based on the sample from $\mathrm{N}(0,1)$
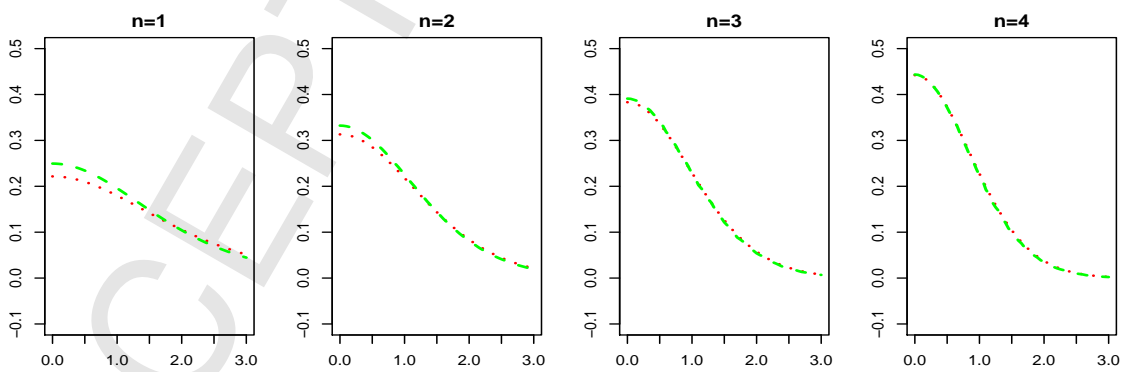

Figure 5: Density of the mean based on sample from logistic $(0,1)$ distribution 

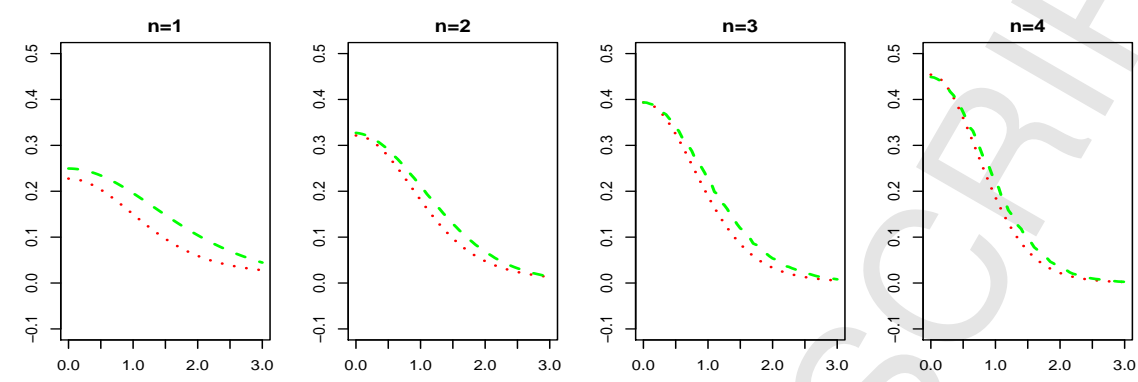

Figure 6: Density of the Huber estimator, $\mathrm{k}=1.4$, sample from logistic $(0,1)$ distribution
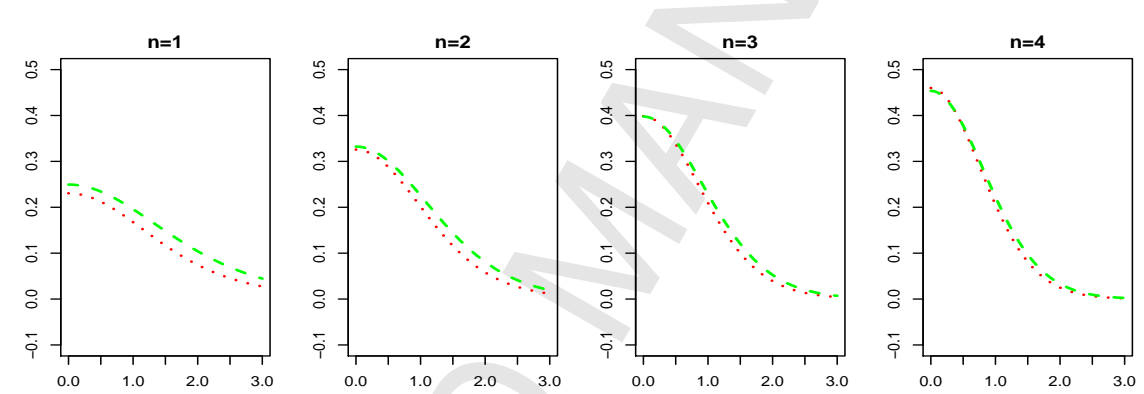

Figure 7: Density of the MLE, sample from logistic $(0,1)$ distribution
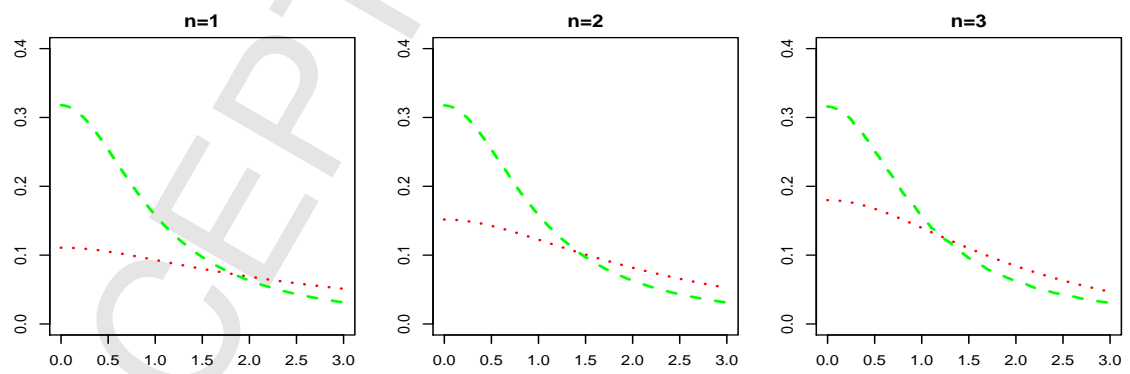

Figure 8: Density of the mean, sample from the Cauchy $(0,1)$ distribution 

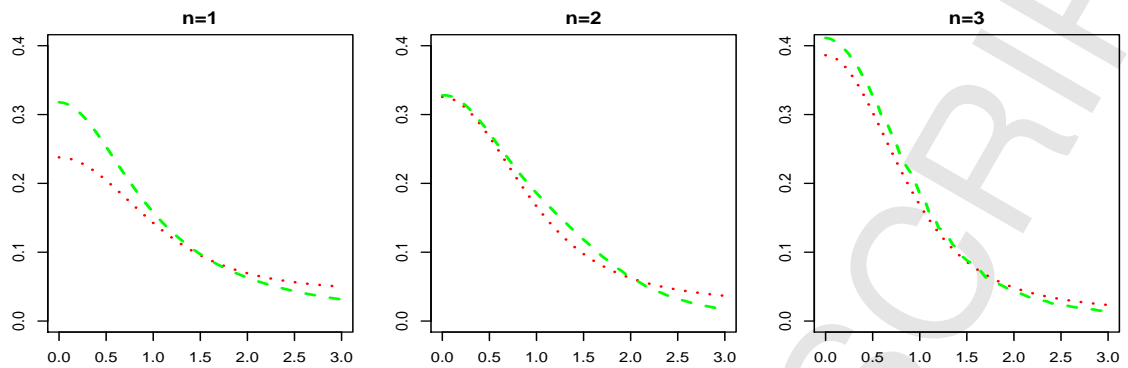

Figure 9: Density of the Huber estimator, $\mathrm{k}=1.4$, sample from the Cauchy $(0,1)$ distribution
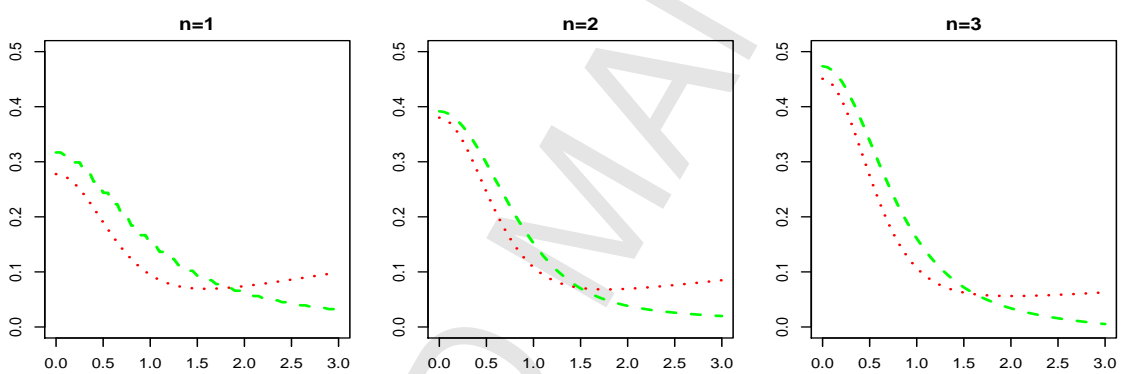

Figure 10: Density of the MLE, sample from the Cauchy $(0,1)$ distribution
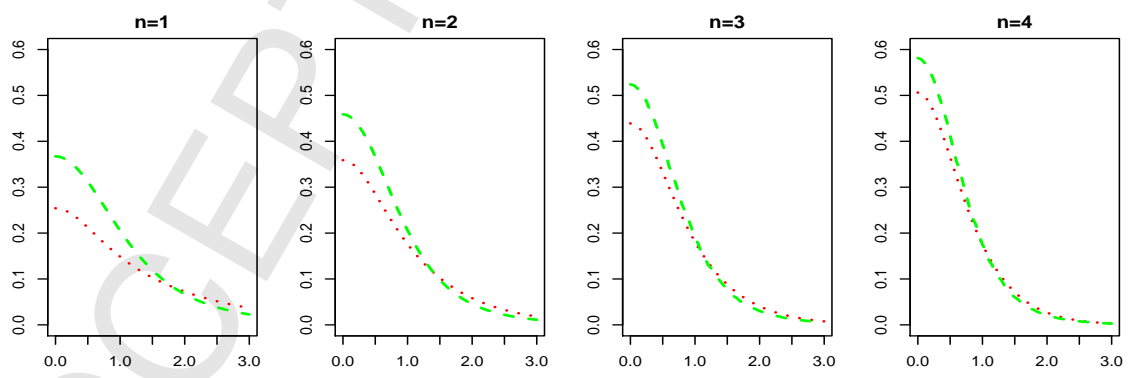

Figure 11: Density of the mean, sample from $t_{3}$ distribution 

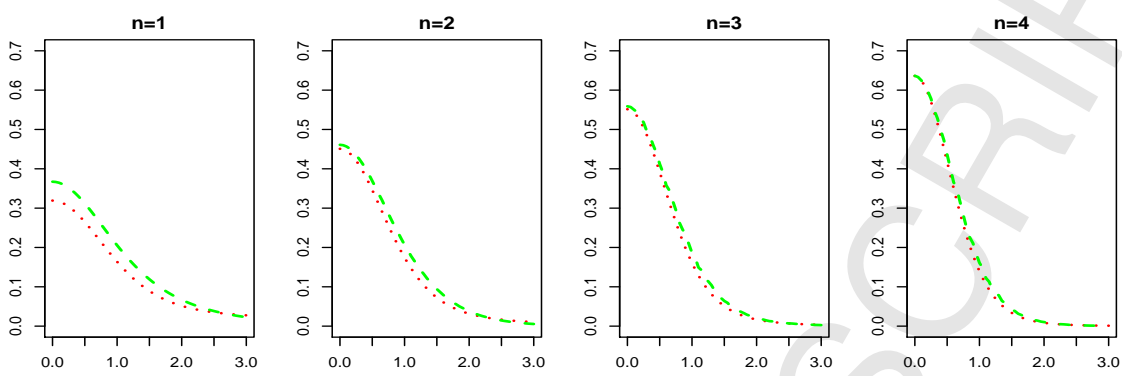

Figure 12: Density of the Huber estimator, $\mathrm{k}=1.4$, sample from $t_{3}$ distribution
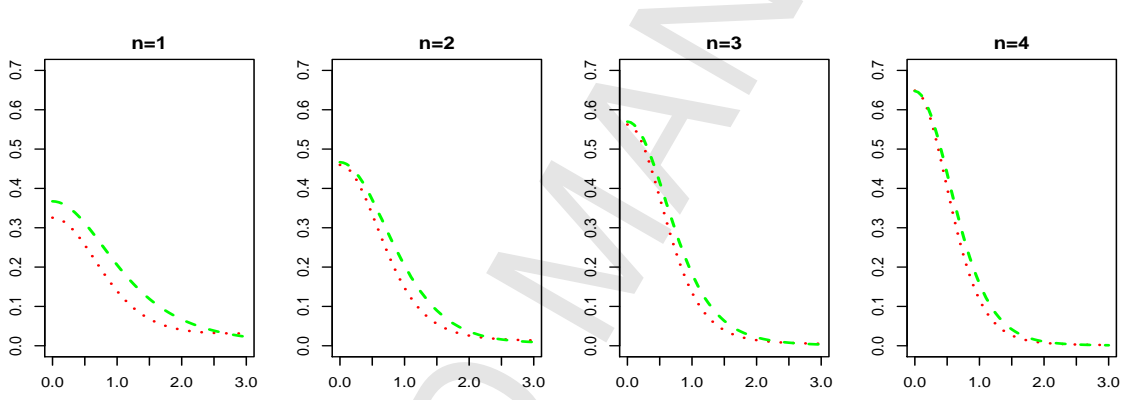

Figure 13: Density of the MLE, sample from $t_{3}$ distribution
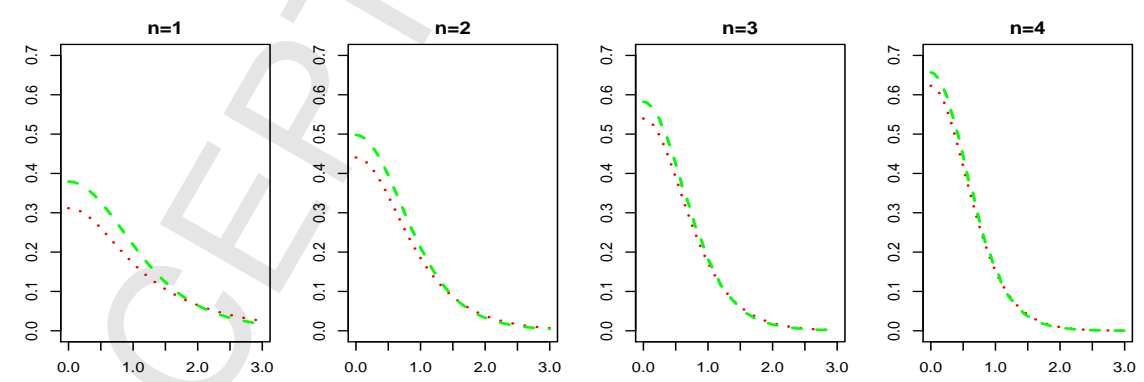

Figure 14: Density of the mean, sample from $t_{5}$ distribution 

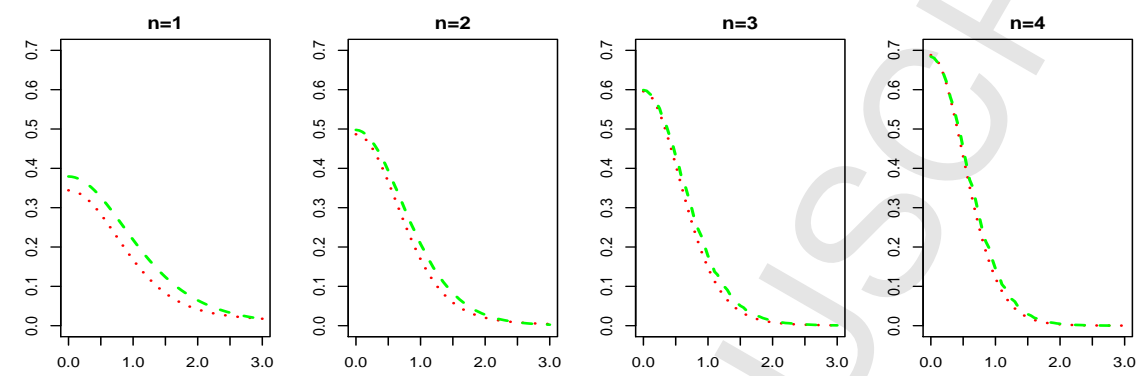

Figure 15: Density of the Huber estimator, $\mathrm{k}=1.4$, sample from $t_{5}$ distribution
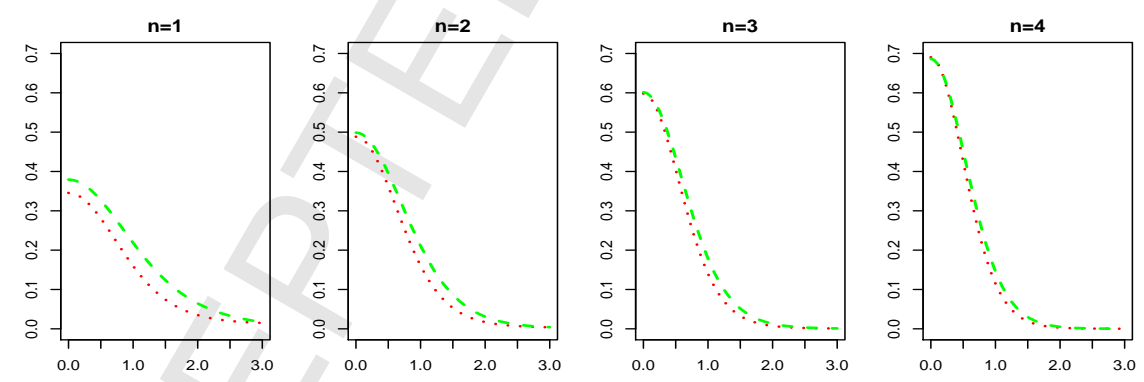

Figure 16: Density of the MLE, sample from $t_{5}$ distribution 


\section{Conclusion}

Both methods lead to similar outcomes for normally distributed data. The results are also quite similar for $f$ logistic unless $t$ is close to 0 . The method based on (2) does not work for the Laplace distribution whose density is not continuously differentiable. As it was already observed by Field and Ronchetti (1990), their approximation does not work very well for the Laplace distribution, either. For the Cauchy distribution the obtained results vary, although the differences between two methods are smaller for $\mathrm{Hu}-$ ber estimator than for the mean. The situation is similar for the Student's t-distribution, although the results improve. It is of interest that the approximations are very close to each other for the maximum likelihood estimators; this apparently demonstrates the important role of the score functions.

Even though formula (2) provides exact expression of the density of the estimator, the discrepancies are caused by approximation of integrals (even resulting in negative values for Laplace distribution). The main disadvantage of this method is its time complexity. Since for larger $n$ the computations take a lot of time, this method proves to be inefficient for large $n$. On the other hand, the time consumed when approximating the density by saddlepoint techniques does not increase with larger $n$.

Although it may seem useless to estimate $f_{n}$ for $n=1$, in the case of mean it is approximating the density $f$ itself, and mainly it shows that these "approximations work well even for $n=1 "$.

\section{Acknowledgement}

The authors thank Elvezio Ronchetti for his interest and for the inspiring discussions on the subject. Also they thank the Editor and the Referee for their consideration and valuable remarks, which considerably helped to improve the readability of the text.

\section{References}

[1] O. E. Barndorff-Nielsen and D. R. Cox. Edgeworth and saddlepoint approximations with statistical applications (with discussion). J. Royal Statist. Soc. B, 41:279-312, (1979).

[2] R. W. Butler. Saddlepoint Approximations with Applications. Cambridge University Press, Cambridge, UK, (2007). 
[3] H. E. Daniels. Saddlepoint approximations on statistics. Ann. Math. Statist., 25:631-650, (1954).

[4] C. A. Field and F. R. Hampel. Small-sample asymptotic distribution of m-estimators of location. Biometrika, 69:29-46, (1982).

[5] C. A. Field and E. Ronchetti. Small Sample Asymptotics. Institute of Mathematical Statistics - Monograph Series, Hayward, CA, (1990).

[6] F. R. Hampel. Some small-sample asymptotics. In J. Hájek, editor, Proc. Prague Symposium on Asymptotic Statistics, pages 109-126. Charles University in Prague, (1973).

[7] S. Huzurbazar. Saddlepoint approximations on statistics. The American Statistician, 53:225-232, (1999).

[8] J. L. Jensen. Saddlepoint Approximations. Oxford University Press, Oxford, UK, (1995).

[9] J. Jurečková. Equivariant estimators and their asymptotic representations. Tatra Mountains Mathematical Publications, 17:1-9, (1999).

[10] J. Jurečková. Finite sample distribution of regression quantiles. Statistics 83 Probability Letters, 80:1940-1946, (2010).

[11] J. Jurečková and X. Milhaud. Derivative in the mean of a density and statistical applications. In C. Léger M. Moore, S. Froda, editor, IMS Lecture Notes, Monograph Series, volume 42, pages 216-230. IMS, Hayward, CA, (2003).

[12] J. Jurečková and P. K. Sen. M-estimators and l-estimators of location: Uniform integrability and asymptotically risk-efficient sequential versions. Sequential Analysis, 1:27-56, (1982). 